\title{
Concordancia entre los criterios diagnósticos RDC/TMD y su actualización DC/TMD, aplicados a la patología inflamatoria de la articulación temporomandibular.
}

\author{
Agreement between Diagnostic Criteria RDC/TMD and its \\ update DC/TMD, applied to the inflammatory pathology of the \\ Temporomandibular Joint.
}

\author{
Gabriela Peña ${ }^{*}$, Walter Díaz², Guillermo Flores², Katina Marinkovic², Fernando Romo², Rolando Schulz².
}

1. Práctica Privada, Santiago, Chile. 2. Departamento de Prótesis, Facultad de

Odontología, Universidad de Chile, Chile.

* Correspondencia Autor: Gabriela Peña Burgos | Dirección: Pirámide 1058, San Miguel, Santiago, Chile | Teléfono: +569 99193775 | E-mail: gpena. odontologia@gmail.com

Trabajo recibido el 28/11/2018.

Aprobado para su publicación el 20/05/2019

\section{RESUMEN}

Objetivo: Evaluarelgrado de concordanciaentrelas prevalencias detrastornosarticulares inflamatorios (TAI) de las Articulaciones Temporomandibulares (ATM), obtenidas con los Criterios Diagnósticos de Investigación para Trastornos Temporomandibulares (RDC/ TMD) y los Criterios Diagnósticos para Trastornos Temporomandibulares (DC/TMD). Materiales y métodos: 59 pacientes adultos chilenos fueron examinados según el Eje I de los RDC/TMD y el Eje I de los DC/TMD para determinar la prevalencia de TAI. Luego se compararon los resultados obtenidos de cada protocolo con el test Kappa de Cohen para evaluar la concordancia entre ambos. Resultados: La prevalencia de TAI para ambos criterios fue de $22 \%$, con una concordancia casi perfecta (kappa=0,91). Al comparar las prevalencias de TAI por articulación, se obtuvo un grado de acuerdo sustancial (kappa $=0,77$ ) para la ATM derecha y un grado de acuerdo casi perfecto (kappa $=0,94$ ) para la ATM izquierda. Al comparar las prevalencias de TAl según género y edad se obtuvo una concordancia estadísticamente significativa en la mayoría de los casos. Conclusión: Hay concordancia estadísticamente significativa entre los datos obtenidos con los RDC/TMD y los DC/TMD, en el diagnóstico de trastornos articulares inflamatorios.

PALABRAS CLAVE

Trastornos articulares inflamatorios; RDC/TMD; DC/TMD; ATM.

Rev. Clin. Periodoncia Implantol. Rehabil. Oral Vol. 12(2); 70-73, 2019.

\section{ABSTRACT}

Aim: To assess the agreement between the prevalence of inflammatory joint disorders (IJD) obtained with Research Diagnostic Criteria for Temporomandibular Disorders (RDC/TMD) and Diagnostic Criteria for Temporomandibular Disorders (DC/TMD). Materials and methods: 59 Chilean adult patients were examined according to Axis I of the RDC/TMD and Axis I of the DC/TMD to determine prevalence of IJD. Then, the results obtained from each protocol were compared with Cohen's Kappa test to assess the agreement between them. Results: The prevalence of IJD for both criteria was $22 \%$, with almost perfect agreement (kappa $=0.91)$. When comparing the prevalences of IJD per joint, a substantial agreement (kappa $=0.77)$ was obtained for the right TMJ and an almost perfect agreement (kappa $=0.94$ ) for the left TMJ. When comparing the prevalences of IJD according to gender and age, a statistically significant agreement was obtained in the majority of cases. Conclusion: There is statistically significant agreement between the data obtained with the RDC/TMD and the DC/TMD, in the diagnosis of inflammatory joint disorders.

\section{KEY WORDS}

Inflammatory joint disorders (IJD); RDC/TMD; DC/TMD; TMJ

Rev. Clin. Periodoncia Implantol. Rehabil. Oral Vol. 12(2); 70-73, 2019. 


\section{INTRODUCCIÓN}

Los Trastornos Temporomandibulares (TTM) son un grupo heterogéneo de condiciones clínicas que involucran a la articulación temporomandibular (ATM), músculos masticadores y estructuras asociadas $^{(1,2)}$ y corresponden a un trastorno complejo resultante de una interacción de causas de múltiples dominios, genéticos y ambientales ${ }^{(3)}$. Los signos y síntomas característicos son el dolor, la limitación funcional y los ruidos articulares ${ }^{(4)}$. El dolor es la razón por la que la mayoría de los pacientes busca tratamiento ${ }^{(2,5,6)}$ y, en especial, cuando es crónico representa una fuente de malestar y grave alteración de la calidad de vida de las personas que lo padecen ${ }^{(2)}$.

Dentro de los TTM más comunes están el dolor miofascial, los desplazamientos discales y la osteoartritis ${ }^{(7)}$.

Los Trastornos Articulares Inflamatorios (TAI), son un subtipo de TTM en los que el dolor y la inflamación están presentes, síntomas limitantes y por los que la mayoría de pacientes buscan y necesitan tratamiento. Dentro de éstos encontramos a la artralgia, la osteoartritis y la osteoartrosis.

A lo largo de los años, la clasificación de los TTM ha sido confusa, debido a la extensa cantidad de taxonomías existentes ${ }^{(2)}$ y han sido escasos los criterios diagnósticos objetivos que puedan diferenciar fácil y claramente a las personas que padecen una condición de las que no(5).

La necesidad de proveer investigaciones clínicas con sistemas estandarizados de examinación, diagnóstico y clasificación para los TTM más comunes permitió el desarrollo de los Criterios Diagnósticos de Investigación para Trastornos Temporomandibulares, conocidos por sus siglas en inglés como RDC/TMD(5). Los RDC/TMD han sido el protocolo de diagnóstico más utilizado para la investigación de $\mathrm{TTM}^{(8)}$.

Aún cuando los RDC/TMD fueron un sistema modelo cuando se publicaron, sus autores reconocieron que era sólo un comienzo y que se necesitaban más investigaciones para mejorar su validez y utilidad clínica. Por lo que, en 2013, se publican los Criterios Diagnóstico para TTM (DC/ TMD), en los que se ha aumentado la sensibilidad y especificidad, siendo apropiados para su uso en ambientes clínicos y de investigación ${ }^{(6)}$. La versión validada al español de los DC/TMD se encuentra en proceso.

El propósito del presente estudio es evaluar el grado de concordancia entre las prevalencias de trastornos articulares inflamatorios (TAI) según ambos protocolos diagnósticos RDC/TMD y DC/TMD.

\section{MATERIALES Y MÉTODOS}

\section{Población de estudio}

Estudio descriptivo, analítico y comparativo, de corte trasversal, realizado en la Facultad de Odontología de la Universidad de Chile. Incluyó a pacientes atendidos en la Clínica Integral del Adulto durante el primer semestre del año 2017. Población de 59 pacientes.

\section{Consideraciones éticas}

El protocolo de estudio se encuentra adscrito al PRI-ODO 012/016 "Análisis in vitro de la resorción ósea inducida en articulaciones temporomandibulares afectadas de osteoartritis", fue revisado y aprobado por el Comité ético-científico de la Facultad de Odontología de la Universidad de Chile (FOUCH). Para participar en el estudio, los pacientes firmaron un consentimiento informado (Anexo1).

\section{Estudio de la muestra}

Se incluyeron a los pacientes asistentes a la Clínica Integral del Adulto (CIA), durante el primer semestre del año 2017, que estuvieran dispuestos a participar comprendiendo y firmando el consentimiento informado de investigación. Se excluyeron a aquellos pacientes con diagnóstico de poliartritis y/o cuadros dolorosos odontogénicos. La muestra constó de 59 pacientes.

\section{Registro de datos}

La información fue obtenida mediante dos exámenes clínicos, cada uno obtenido de los protocolos de examen físico correspondientes al Eje I de los RDC/TMD y al Eje I de los DC/TMD, respectivamente; ambos disponibles en la página del Consorcio Internacional RDC-TMD (https:// ubwp.buffalo.edu/rdc-tmdinternational/).

Se calibró 1 examinador para realizar los exámenes clínicos de análisis de TTM por 1 profesional especialista en TTM, siguiendo las Guías para la calibración y capacitación del Consorcio Internacional RDC-TMD, para cada protocolo RDC/TMD y DC/TMD, también disponibles en la página del Consorcio Internacional RDC-TMD (https://ubwp.buffalo.edu/rdctmdinternational/).

Los resultados para cada examen fueron analizados, a través de algoritmos ya establecidos, obteniéndose el diagnóstico de TTM Articular Inflamatorio para cada paciente según RDC/TMD y DC/TMD. Los diferentes diagnósticos se presentan en la Tabla 1.

Luego, las prevalencias de TAI para cada protocolo fueron comparadas usando el test Kappa de Cohen para evaluar la concordancia entre ellos.

Tabla 1: Diagnósticos Articular Inflamatorios según RDC/TMD y DC/TMD

\begin{tabular}{l|l}
\hline \multicolumn{1}{c|}{ Diagnóstico RDC/TMD } & \multicolumn{1}{|c}{ Diagnóstico DC/TMD } \\
\hline - Sin Diagnóstico & • Sin Diagnóstico \\
\hline - Artralgia & • Artralgia \\
\hline - Osteoartrosis & $\bullet$ Trastorno Articular Degenerativo \\
\hline - Osteoartritis & \\
\hline
\end{tabular}

\section{Análisis estadístico}

Diagnósticos de TTM de tipo articular e inflamatorio: en el nuevo protocolo DC/TMD ambos términos Osteoartrosis y Osteoartritis, al no ser utilizados de forma sistemática en medicina, se subclasifican bajo el término más amplio "Trastornos Articulares Degenerativos" (TAD), el uso de este término más amplio está respaldado por la Asociación Americana de Cirujanos Orales y Maxilofaciales ${ }^{(6)}$. Para DC/TMD, los diagnósticos Artralgia y TAD pueden coexistir, no obstante para motivos de este estudio, y para hacer el análisis, aquellos pacientes que tenían ambos diagnósticos se clasificaron sólo en TAD; por lo que para cada articulación, derecha e izquierda, hay 3 diagnósticos posibles, estos son Sin diagnóstico [0], Atralgia [1] y Osteoartrosis/Osteoartritis/TAD [2], considerando a un "individuo enfermo" a aquel que tenga por lo menos una de las articulaciones, derecha o izquierda, con alguno de estos diagnósticos [1] ó [2], llamados en conjunto como Trastornos Articulares Inflamatorios (TAI).

\section{- Género: Femenino [0], Masculino [1]}

- Grupos etarios: Se subclasificaron en 4 grupos:

- 18 a 24

- 25 a 44

- 45 a 59

• 60 ó más

Los resultados fueron analizados como variables de prevalencia, en forma descriptiva (tasas, gráficos y tablas).

Las prevalencias de TAl obtenidas según cada protocolo RDC/ TMD y DC/TMD fueron comparadas entre sí usando el test Kappa de Cohen, para obtener el grado de concordancia; siendo estadísticamente significativo un kappa > 0,6; estableciéndose como buena concordancia o grado de acuerdo sustancial (kappa $=0,61-0,8)$ o grado de acuerdo o concordancia casi perfecta $(\mathrm{kappa}=0,81-1)^{(9)}$.

\section{RESULTADOS}

El rango de edad de los pacientes examinados osciló entre 19 y 80 años, con un promedio de edad de 53,68 años, de los cuales $69,49 \%$ fueron mujeres $(n=41)$ y $30,51 \%$ fueron hombres $(n=18)$. El mayor número de participantes examinados estuvo en el rango de 45 a 60 años $(49,15 \%)$.

La prevalencia de participantes que presentó al menos un diagnóstico de TTM, diagnosticado por al menos uno de los dos criterios RDC/TMD y/o DC/TMD fue de $57,6 \%(n=34)$. La prevalencia según RDC/TMD fue de $52,54 \%(n=31)$ y de $D C / T M D$ de $56 \%(n=33)$. El grado de acuerdo entre los criterios RDC/TMD y DC/TMD fue casi perfecto (kappa 0,83). Hay una mayor proporción de TTM en mujeres con un $61 \%$ versus el $50 \%$ de los hombres.

La prevalencia de TAl, según los RDC/TMD fue de 13,56\% para Artralgia $(n=8), 3,38 \%(n=2)$ para Osteoartritis y $5,08 \%(n=3)$ para Osteoartrosis, y según los DC/TMD fue de $12 \%(n=7)$ para Artralgia y $10 \%(n=6)$ para TAD, con una prevalencia total de $22 \%$ para ambos protocolos y una concordancia casi perfecta (kappa 0,91), su distribución según género y edad se muestra en la Tabla 2 y el grado de concordancia, según las variables en estudio se ve reflejado en la Tabla 3.

\section{DISCUSIÓN}

A lo largo de los años, la clasificación de los TTM ha sido confusa, debido a la extensa cantidad de taxonomías existentes ${ }^{(2)}$. La necesidad de proveer investigaciones clínicas con sistemas estandarizados de examinación, diagnóstico y clasificación para los TTM más comunes permitió el desarrollo de los Criterios Diagnósticos de Investigación para Trastornos Temporomandibulares (RDC/TMD) ${ }^{(10,11)}$. 
Tabla 2: Prevalencia de TAI por articulación y género, según RDC/TMD y DC/TMD

\begin{tabular}{lc|c|c|c|c|c|c|c}
\hline & \multicolumn{2}{c|}{$\begin{array}{c}\text { ATM } \\
\text { derecha } \\
(\%)\end{array}$} & \multicolumn{2}{c|}{$\begin{array}{c}\text { ATM } \\
\text { izquierda } \\
(\%)\end{array}$} & \multicolumn{2}{c|}{$\begin{array}{c}\text { Mujeres } \\
(\%)\end{array}$} & \multicolumn{2}{c}{$\begin{array}{c}\text { Hombres } \\
(\%)\end{array}$} \\
\cline { 2 - 10 } & RDC & DC & RDC & DC & RDC & DC & RDC & DC \\
\cline { 2 - 10 } Artralgia & 11,86 & 10,17 & 10,17 & 8,47 & 17,07 & 17,07 & 5,55 & 0 \\
\hline Osteoartritis & 1,69 & & 1,69 & & 4,88 & & 0 & \\
\hline Osteoartrosis & 1,69 & & 5,09 & & 4,88 & & 5,55 & \\
\hline TAD & & 8,47 & & 6,78 & & 12,19 & & 5,55 \\
\hline
\end{tabular}

Tabla 3: Grado de concordancia entre RDC/TMD y DC/TMD

\begin{tabular}{|c|c|c|c|}
\hline \multirow[b]{3}{*}{ TTM } & \multicolumn{2}{|c|}{ Prevalencia (\%) } & \multirow{2}{*}{$\begin{array}{c}\text { Concordancia } \\
{\text { (kappa })^{*}}^{*}\end{array}$} \\
\hline & RDC/TMD & DC/TMD & \\
\hline & 52,54 & 56 & 0,83 \\
\hline TAI & 22 & 22 & 0,91 \\
\hline TAI x ATM der & 15,25 & 18,64 & 0,77 \\
\hline TAl x ATM izq & 16,95 & 15,25 & 0,94 \\
\hline TAl $x$ mujeres & 27,9 & 29,3 & 0,94 \\
\hline TAI $x$ hombres & 11,12 & 5,6 & 0,65 \\
\hline TAl x $18-24$ años & 75 & 50 & 0,5 \\
\hline TAl $\times 25-44$ años & 50 & 50 & 1 \\
\hline TAl x $45-59$ años & 20,7 & 20,7 & 0,8 \\
\hline TAI $x \geq 60$ años & 0 & 0 & - \\
\hline
\end{tabular}

La actualización de los RDC/TMD a DC/TMD, además de ser necesaria para su uso en ambientes clínicos, con un aumento en su validez diagnóstica, va acorde al mejor entendimiento de la problemática que rodea a los TTM, que sería la presencia de dolor, característica más invalidante y por la que la mayoría de pacientes busca tratamiento(2,5,6), por lo que se hace innegable la necesidad de un protocolo diagnóstico cuyo enfoque principal sea este síntoma.

El dolor ha ido ganando espacio como eje central de los TTM, lo que se ve reflejado en la nueva clasificación DC/TMD, dejando un diagnóstico articular, Artralgia, en el capítulo de dolor, junto a otros TTM dolorosos como la Mialgia Local y el Dolor Miofascial. Por otra parte, los procesos inflamatorios y degenerativos agrupados en los diagnósticos de Osteoartritis y Osteoartrosis en los RDC/TMD, tienden a confundir en la práctica clínica, especialmente cuando diagnosticamos Artralgia y Artritis de forma separada. Por lo tanto, para asegurar la sensibilidad y especificidad en el diagnóstico, el uso del término Trastornos Articulares Degenerativos ayuda tanto al registro clínico como de investigación. Se puede decir que en promedio, los TTM afectan entre un 40 y $50 \%$ de la población general(12), en Chile no existen datos al respecto. El porcentaje de pacientes que presentan TTM es muy alto, siendo menor quienes requieren tratamiento. Dentro de los pacientes que requieren tratamiento, se encuentran aquellos que presentan TTM asociados a dolor e inflamación, como es el caso de los TAI.
Numerosos estudios epidemiológicos chilenos han examinado la prevalencia de algún subgrupo de TTM en poblaciones específicas ${ }^{(11,}$ 13-17). Estos trabajos tienen en común la examinación bajo un protocolo estandarizado ya sea RDC/TMD o DC/TMD y la prevalencia de TTM varía entre 25 y $53 \%$.

Estas variaciones en la prevalencia de TTM se deben a las diferencias en las muestras de estudios, ya que al ser poblaciones específicas no reflejan la prevalencia real de la población chilena.

En la actualidad, los estudios que hay con el nuevo protocolo DC/ TMD son escasos y la mayoría de origen transversal en poblaciones determinadas ${ }^{(14,18-21)}$. Aún cuando los DC/TMD se publicaron en 2013, y expertos en el tema consideran a los RDC/TMD como obsoletos, aún hay estudios con data posterior a esta que usaron como criterios diagnósticos los antiguos $\mathrm{RDC} / \mathrm{TMD}^{(16,17,22)}$, esto debido a que, a la fecha, aún no está disponible la versión traducida al español validada por el Consorcio Internacional RDC-TMD.

Los datos de prevalencia encontrados en este estudio son similares a los reportados en estudios realizados en la Clínica Integral del Adulto de la FOUCH, entre años 2005 y $2007^{(23-25)}$, donde la prevalencia general de TTM varía entre $55 \%$ y $58 \%$ y la prevalencia de TAl es cercana al $20 \%$, siendo más afectadas mujeres que hombres. En estos estudios se usaron los RDC/TMD y, al igual que en este trabajo, se evaluaron más mujeres que hombres.

Al comparar la prevalencia de Trastornos Articulares Inflamatorios según ambos criterios RDC/TMD y DC/TMD hay concordancia estadísticamente significativa (kappa $>0,6$ ) siendo el grado de asociación en la mayoría de los casos casi perfecto (kappa>0,8); excepto en el grupo etario entre 18 y 24 años, donde si bien hubo concordancia, ésta no era estadísticamente significativa debido al bajo $\mathrm{N}$.

Dentro de los TAl, el diagnóstico más prevalente fue Artralgia y el género más afectado fue el femenino; datos comparables a otros estudios en población chilena ${ }^{(11,13,23-25)}$.

En un estudio el 2012, Diaz y cols. ${ }^{(13)}$ determinaron en una población chilena, que el $41,2 \%$ de la población estudiada presentaba un diagnóstico que requería tratamiento, dentro de los que se encontraba la osteoartritis, por la presencia de dolor e inflamación. Para tratar a los pacientes con TTM, generalmente se inicia con la utilización de métodos conservadores reversibles ${ }^{(13)}$, sin embargo hay un porcentaje de pacientes que no responde a estas terapias menos invasivas, obligando al clínico a buscar otras alternativas de tratamiento, siendo de mayor utilidad un diagnóstico certero y a tiempo, que ayude al clínico en la búsqueda del mejor tratamiento posible.

Cabe destacar que dentro de las limitaciones de este estudio, está la falta de la traducción validada en español de los DC/TMD, por lo que se comparó un criterio válido y estandarizado, RDC/TMD, con una traducción no validada por el Consorcio Internacional RDC-TMD de DC/ TMD. Se espera que cuando salga la traducción validada al español de los DC/TMD se pueda replicar este estudio.

\section{CONCLUSIONES}

1. Hay concordancia estadísticamente significativa entre los RDC/ TMD y DC/TMD en el diagnóstico de trastornos articulares inflamatorios.

2. Los resultados obtenidos permiten contribuir al conocimiento y uso de los DC/TMD como instrumento diagnóstico de los trastornos articulares inflamatorios.

3. Este es un primer paso hacia la aseveración que los resultados obtenidos en los últimos años con los RDC/TMD son comparables con los que se obtienen en la actualidad usando los DC/TMD.

\section{CONFLICTO DE INTERÉS}

Los autores de este estudio no presentan conflictos de intereses. 


\section{Bibliografía}

1. Griffiths R. Report of the president's conference on examination, diagnosis and management of temporomandibular disorders. J Am Dent Assoc. 1983;106:75-77.

2. Romo F, Díaz W, Schulz R, Torres M. Tópicos de odontología integral. Santiago: Universidad de Chile, Facultad de Odontología, 2011

3. Slade GD, Ohrbach R, Greenspan JD, Fillingim RB, Bair E, Samders AE, et al. Painful temporomandibular disorder: Decade of discovery from OPPERA Studies. $J$ Dent Res. 2016;95:1084-1092.

4. Taboada AO, Gómez GY, Taboada AS, Mendoza NV. Prevalencia de signos y síntomas de los trastornos temporomandibulares en un grupo de adultos mayores. ADM. 2004;61:125-129.

5. Dworkin SF, LeResche L. Research diagnostic criteria for temporomandibula disorders: Review criteria, examinations and specifications, critique. J Craniomandib Disord. 1992;6:301-355.

6. Schiffman E, Ohrbach R, Truelove E, Look J, Anderson G, Goulet JP, et al. Diagnostic criteria for temporomandibular disorders (DC/TMD) for clinical and research applications: Recommendations of the International RDC/TMD Consortium Network and Orofacial Pain Special Interest Group. J Oral Facial Pain Headache. 2014;28:6-27.

7. Gauer RI, Semidey MJ. Diagnosis and treatment of temporomandibular disorders. Am Fam Physician. 2015;91:378-386.

8. Schiffman E, Truelove E, Ohrbach R, Anderson G, John M, List T, et al. Assessment of validity of research diagnostic criteria for temporomandibular disorders: Overview and methodology. J Orofac. Pain. 2010;24:7-24.

9. Abraira V. El índice kappa. SEMERGEN. 2001;27:247-249.

10. Dworkin SF. Research diagnostic criteria for temporomandibular disorders: current status \& future relevance. J Oral Rehabil. 2010;37:734-743.

11. Schulz R, Moya M, Carmen R, Ivanovic M, Diaz M. Relación entre los diagnósticos del eje I (físico) y el eje II (psicosocial), según los criterios diagnósticos de investigación para trastornos temporomandibulares (RDC/TMD), en una población chilena. Rev Dental Chile. 2011;102:24-29

12. Nilsson I, List T, Drangsholt M. Prevalence of temporomandibular pain and subsequent dental treatment in Swedish adolescents. J Orofac Pain . 2005:19:144-150. 13. Díaz W, Guzmán CL, Martín C. Prevalencia y necesidad de tratamiento de trastornos temporomandibulares en una población chilena. Rev. Arch med. 2012;16:602-609.

14. Gatica P, Ramos G. Prevalencia de trastornos temporomandibulares relacionados con dolor y cefalea, en una población adolescente de la comuna de Talca. Estudio piloto. Trabajo de Investigación requisito para optar al título de Cirujano Dentista. Universidad de Talca. 2014.
15. Sandoval I, Ibarra N, Flores G, Marinkovic K, Díaz W, Romo F. Prevalencia de trastornos temporomandibulares según los CDI/TTM, en un grupo de adultos mayores de Santiago, Chile. Int. J Odontostomat. 2015;9:73-78

16. Aravena PC, Arias R, Aravena-Torres R, Seguel-Galdames F. Prevalencia de trastornos temporomandibulares en adolescentes del sur de Chile, año 2015. Rev Clin Periodoncia Implantol Rehabil Oral. 2016;9:244-252.

17. Jimenez-Silva A, Peña C, Lee-Muñoz X, Vergara-Nuñez C, Tobar-Reyes J, Frugone-Zambra R. Patología temporomandibular asociada a masticación unilateral en adultos jóvenes. Rev. Clin Periodoncia Implantol Rehabil Oral. 2016;9:125-131.

18. Graue AM, Jokstad A, Assmus J, Skeie MS. Prevalence among adolescents in Bergen, Western Norway, of temporomandibular disorders according to the DC/TMS criteria and examination protocol. Acta Odontol Scand. 2016;74:449-455.

19. Murrieta J, Alvarado E, Valdez M, Orozco L, Meza J, Juárez ML. Prevalence of temporomandibular joint disorders in a Mexican elderly group. J Oral Res. 2016;5:13-18

20. Jivnani HM, Tripathi S, Shanker R, Singh BP, Agrawal KK, Sighal R. A study to determine the prevalence of temporomandibular disorders in a young adult population and its association with psychological and functional occlusal parameters. J Phosthodont. 2019;28(1):e445-e449.

21. Lövgren A, Österlund C, llgunas A, Lampa E, Hellström F. A high prevalence of TMD is related to somatic awareness and pain intensity among healthy dental students. Acta Odontol Scand. 2018;18:10-17.

22. Amaya SY, Casanova CM, Barrera JP, Benavides RA, Sánchez NY, Buriticá A Prevalencia de trastornos de la articulación temporomandibular según los criterios diagnóstico de investigación en pacientes preortodónticos. Univ Odontol. 2014;33:19-28

23. Ávila D. Prevalencia de trastornos temporomandibulares articulares y su relación con la pérdida de soporte oclusal posterior unilateral en adultos. Trabajo de Investigación requisito para optar al título de Cirujano Dentista. Universidad de Chile. 2005

24. Bastías V. Prevalencia de trastornos temporomandibulares articulares y su relación con la ausencia de guía anterior en adultos. Trabajo de Investigación requisito para optar al título de Cirujano Dentista. Universidad de Chile. 2006

25. Rojas A. Prevalencia de trastornos temporomandibulares articulares y su relación con la presencia de dolor agudo y crónico en la Clínica Integral los años 2002, 2003, 2004 y 2006. Trabajo de Investigación requisito para optar al título de Cirujano Dentista. Universidad de Chile. 2007. 